\title{
Evaluating the Utility of Fiber Optic Analysis for Dissolution Testing of Drug Products
}

\author{
Christopher A. Martin \\ Technical Training Specialist \\ Varian, Inc., Dissolution Systems \\ 13000 Weston Parkway, Cary, NC 27513
}

T he automation of dissolution testing almost became a necessity in the 1970s, with the onset of regulatory requirements insisting on a performance check for solid dosage forms. Intensive manual labor, long turnaround times from run to run, analyst-to-analyst variability in sampling and filtering, and current 21CFR Part 11 requirements have all been driving the dissolution world's movement toward some sort of automated technique. To date, the dissolution method can be automated through a variety of testing stages or as a total package. Available systems can remove and filter accurate sample aliquots and hold them for further analytical evaluation. Other systems use a closed circuit of fluid flow that allows samples to be removed from the vessels, analyzed via online UV spectroscopy and deposited back into the vessels to alleviate any lost sample volume found with manual methods. Furthermore, additional equipment added to the dissolution system can produce automated LC analysis of dissolution samples or a complete system that will clean vessels between runs, add fresh deaerated media, and start a new dissolution test automatically. Yet the most exciting trend of automation for the dissolution test and what many consider the future of this field is in situ fiber optic sample measurements.

Fiber optic dissolution was first introduced on the market in the late 1990s and is still a relatively

Figure 1: A fiber optic probe allows measurements of dissolved product contents directly in the dissolution vessel. The probes come in a variety of shapes, sizes and orientations depending on the vendor source.

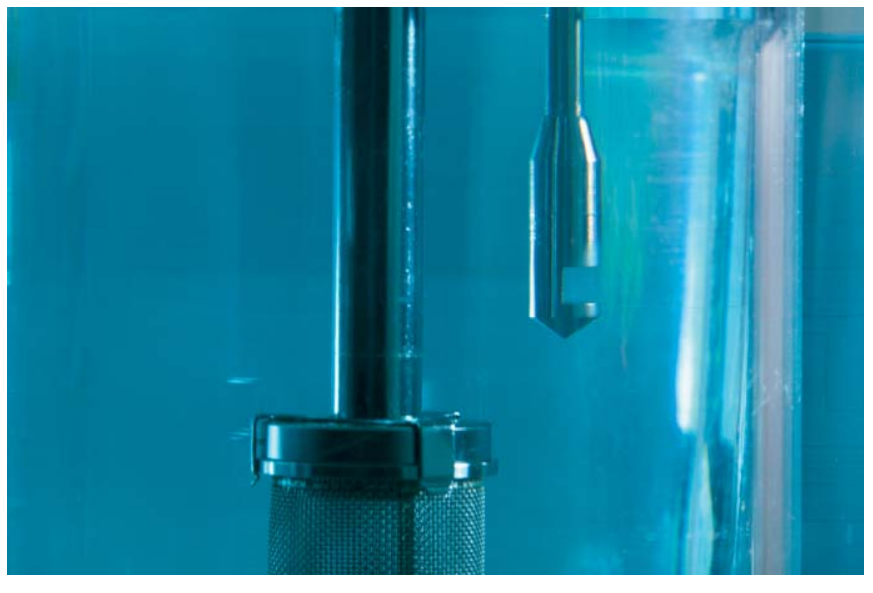

young science. The use of fiber optics allows the direct measurement of dissolved drug in the dissolution vessel via an individual probe for each dosage unit tested (Figure 1). The systems essentially "pump" light from a spectrophotometer to the vessel and then carry the light back to the spectrophotometer where absorbance is measured. No tubes are required to carry sample solutions from one place to another. No filters are necessary to filter the samples. This not only cuts back on consumable materials needed for each test, but also minimizes the amount of effort and time required to validate, when it is not necessary to investigate analyte adsorption, leachability of tubing components, or the effectiveness of the tube cleaning procedure between dissolution runs. In situ measurements also mean that no analyte and sample volumes are removed from the vessels during the test, eliminating the need for complicated calculations to account for those vessel changes. Furthermore, dissolution profiles are calculated in real time and provide instantaneous information on the performance of the product.

Most important, however, is the volume of data that one can acquire from such automation. The amount of time required to perform a full measurement of all vessels during a dissolution test at each timepoint is extremely short and permits time intervals of less than or equal to 1 minute between sample timepoint readings. This means that at least three to four times as many sample timepoints can be measured on a fiber optic system as can be measured on a manual or sample-pumping system. The resulting data provides more accurate information on the true dissolution profile of a solid dosage form (Figure 2). With more accurate data, better in vitro-in vivo correlation and bioequivalence information is available, and will help to streamline the development process, minimize stress on the quality control environment, and result in a cheaper, superior drug product.

Unfortunately, though, the world of pharmaceutical analysis is not always clear-cut and straightforward. Despite the superior advantages provided by the use of fiber optic systems over many other manual and automated techniques, not every drug product is suitable for such analysis. Furthermore, 


\section{Evaluating the Utility ... continued}

Figure 2: Fiber optic dissolution allows for the acquisition of considerably more data than can be collected via other automated or manual techniques. This allows for more accurate dissolution profile determination.

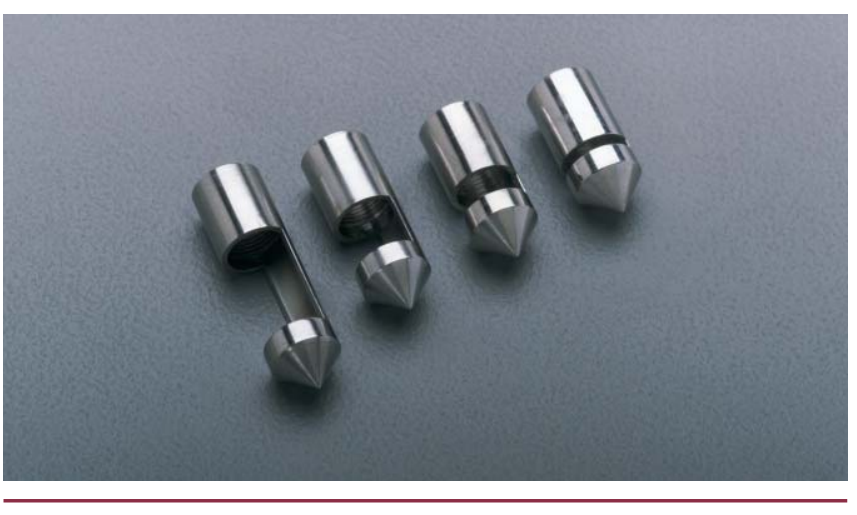

the user of fiber optic systems carries the burden of proving that products analyzed with fiber optics do indeed work with these systems. A little effort and careful consideration of certain aspects of the drug product can simplify evaluating their testing with fiber optic systems.

Probably the most important drug product characteristic to evaluate is the spectrum of each of the drug product's components. The information gained from this evaluation will often be enough to indicate whether or not fiber optic analysis will work for the given product. Before continuing, however, it is important to note that fiber optic analysis is not effectively different than normal UV spectroscopy. The physics are essentially the same. If a sample pathlength of $10 \mathrm{~mm}$ is needed on a typical spectrophotometer, that same pathlength will generally still be needed on a fiber optic system. If an excipient component interferes with the wavelength of maximum absorbance of the API (active pharmaceutical ingredient) on a typical spectrophotometer, it is going to interfere in the same manner with fiber optics. The only real difference between the two systems is how the light gets to the sample. On a typical spectrophotometer, the user places the sample directly in the light path between the lamp and the detector. With fiber optic systems, the user employs a fiber optic probe to carry the light from the lamp to the sample and then carry the non-absorbed light back to the detector. In either case, the outcome is essentially the same: Each system shines light through a sample and onto a detector which measures how much light has been absorbed at specific wavelengths.

With that in mind, individual scans of the spectra of the API and the drug excipient materials should be acquired. The API and excipients should be dissolved or "extracted" in the dissolution medium of choice and should be at concentration levels that mimic those expected during the dissolution test. For capsule formulations, the gelatin shell should be included with the excipient material. These spectra can either be acquired on a fiber optic dissolution system or on a typical spectrophotometer. The similarities of the two systems allow for the assessment of drug characteristics without purchasing the fiber optic system up front. Evaluation of the spectrum will optimally show a peak or two of maximum absorbance for the API while the excipients display neither interfering peaks nor change in baseline attitude at wavelengths corresponding to the absorbance maxima of the API. In this ideal situation, the lack of interference permits the testing of that product with fiber optics. However, in those few cases where an interfering peak or absorbance shift exists between the API and the excipients, special software algorithms may be used to correct for that interference. In most cases, this is performed mathematically using second derivative calculus. While difficult to validate, this approach can be quite useful and will increase the merit of the fiber optic system for evaluating the drug product. Otherwise, the drug product is not appropriate for fiber optic dissolution on systems that lack the algorithms to correct for the interferences.

The spectrum of the API will also provide important insight on the magnitude of the expected absorbance for the samples during the dissolution test. There are two points to look for here. First, the absorbance of the fully released sample must be within the photometric range of the spectrophotometer. If multiple strengths of the sample are to be tested, each of the strengths should be evaluated. If possible, it is useful to perform this measurement on the fiber optic system itself since the different fiber optic spectrophotometers on the market have different photometric range maxima, from approximately 1.5 to $2.5 \mathrm{AU}$. Additionally, the transmittance ability of the probes differs substantially between the major systems. This affects the background absorbance of the system and will add to the total absorbance being measured when probes of weaker transmittance are used. The cumulative effect may be a smaller working dynamic range than that claimed by the manufacturers of the spectrophotometer. If a fiber optic system is not available, typical photometric range data for the available systems should provide enough information to guess on the suitability of the fiber optics for that product. However, it is highly recommended that the fiber optic system be used for this evaluation when possible. If the highest absorbance is outside the range of the fiber optic system, a change in sample pathlength may be needed. This is easily performed on some systems but may be a costly alternative on others (Figure 3 ).

Along with the determination of the highest absorbance expected during the dissolution test, it is also essential to

See Evaluating the Utility ... continued on page 40 


\section{Evaluating the Utility ... continued}

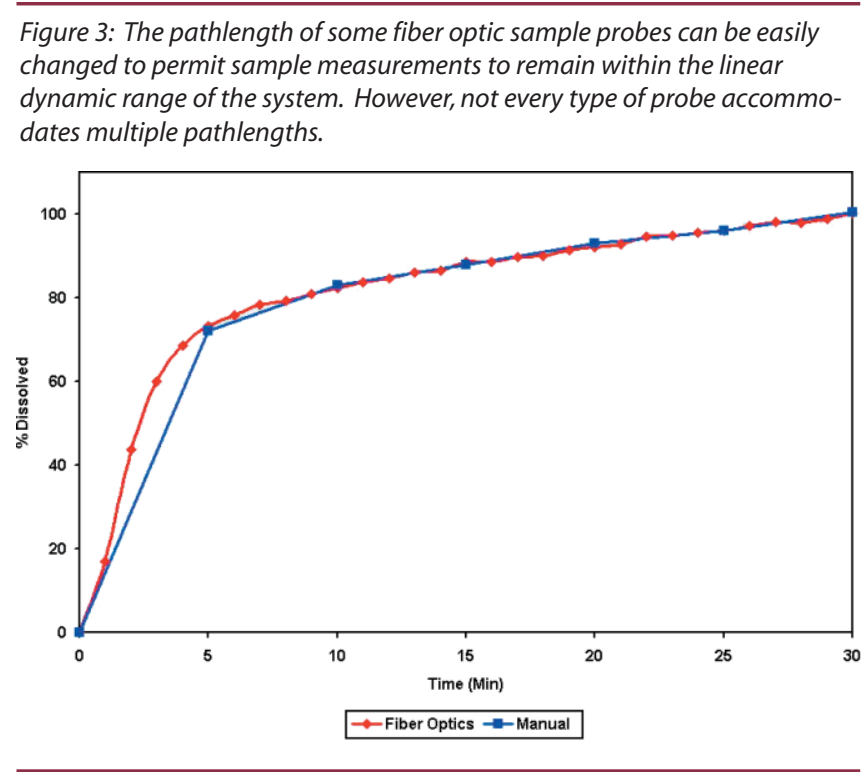

evaluate the lowest absorbance that must be measured. Typically, this is an issue only when specifications for the drug product require sample measurement at timepoints where only 5 to $20 \%$ of the drug is dissolved or released. For analytes that have very weak chromophores, absorbances at these timepoints may be extremely low and could fail to meet the limit of detection of the fiber optic system. Detection limits for fiber optic systems are generally a little higher than the limits determined for the spectrophotometers alone, since the lack of full transmittance of the fiber optic probes adds noise to the measurements. In cases where the LOD is too high for the drug product in question, sample pathlength change (to a longer pathlength) could be considered. Again, this could cause problems with some systems and might facilitate the use of another type of analysis.

After evaluation of the drug component spectra, the physical dissolution process of the drug product should be visually monitored. The relative amount of undissolved material and the pattern of its movement throughout the vessel during the test should be noted. As previously mentioned, sample measurements are taken by the fiber optic system directly in the vessel without filtration. If undissolved particles, typically from excipient materials, move throughout the vessel, those particles may block light being shined through the sample solution at the time of measurement. At moderate turbidity levels, most systems have the capability of mathematically correcting for anything that may provide absorbance (lack of returned light) other than the analyte of interest. However, the blocked light will add to the total baseline absorbance of the system and essentially decrease the linear dynamic range of the system to the point where the absorbance contributed by the undissolved excipients cannot be separated from that caused by the dissolved analyte. Thus, the more circulating particles, the greater the chance of exceeding the total working dynamic range of the system, resulting in a higher probability of measurement issues during the dissolution run. Meanwhile, products with a low proportion of excipient materials, or with materials that settle to the bottom of the vessel during the test, should present few problems for fiber optic analysis.

Evaluation of the drug product component spectra and potential excipient effects will go a long way toward providing sufficient proof of usability of fiber optic dissolution for a particular product and can be partly performed in the absence of the fiber optic system itself. Yet, the effect of changed hydrodynamics in the dissolution vessel must also be evaluated. In a majority of the fiber optic systems on the market, the fiber optic probes reside in the dissolution vessel during the entire dissolution test. However, the addition of any outside structure inside the moving liquid of a hydrodynamically controlled environment will affect the fluid flow patterns. These hydrodynamic changes can affect the dissolution characteristics of certain drug products. While several probe manufacturers have attempted to reduce the hydrodynamic impact of their probes by streamlining their shape and size, the probes still change the flow patterns of the media around the drug product. In cases where the probes must remain resident in the vessel during the dissolution run, it is imperative that manual dissolution studies be performed and compared with fiber optics studies to assess the effect of the hydrodynamic change on the dissolution rate of the drug product. In order to use fiber optic analysis for that drug product, the effect must be minimal.

Despite its technological youth, fiber optic dissolution has already become an important tool for research and development scientists and is beginning to appear in the quality control area of pharmaceutical testing. Its ease of use, high data collection speed and hands-off approach make this the wave of the future for dissolution testing. A little research, minor lab work, and a basic understanding of what to look for can set any chemist in the right direction toward using this up-and-coming technology for their solid dosage products. 